\title{
Performance Analysis of Angle of Arrival Estimation Algorithms for Smart Antenna in Wireless Sensor Networks: An Optimization Approach
}

\author{
N. G. Palan \\ Assistant Professor, E \& TC \\ Department, Cummins College \\ of Engineering For Women, \\ Pune. \\ Research Scholar, Shivaji \\ University, Kolhapur
}

\author{
B. V. Barbadekar \\ Research Guide \\ Shivaji University
}

\author{
Suhas S. Patil, PhD \\ Associate Professor, \\ Electronics Department, \\ K. B. P. College of \\ Engineering, \\ Satara
}

\begin{abstract}
WSN (Wireless Sensor Network) has energy constraints. Main energy consumption is at the (Tx) transmitter/ receiver (Rx) side, which is proportional to data/command packets /frames. Failure at the receiving end asks for re-transmission leading to more power consumption. WSN follows structured layers like medium access control (MAC), physical layer (PHY), link layer etc. PHY deals with RF transmission including antenna. In this paper the focus is given on smart antenna as, WSN deals with systems which need to be adaptive, especially in unknown time varying scenarios. With data transmission; location positioning (localization) and efficient routing are the factors to be considered. Localization of node is done using range measurements which include received signal strength, time of arrival, time difference of arrival (TDOA) and angle-of-arrival (AoA) measurement. By improvising antenna performance i.e. small beam width, we can achieve less erroneous data reception, leading to less energy consumption. In this work, we propose a method based on Non-dominated Sorting Genetic Algorithm (NSGA-II) for localization of nodes using smart antennas. Comparative analysis of its performance is done with different algorithms like LMS (Least Mean Square), LMS and PSO (Particle Swarm Algorithm) for varying number of elements and spacing between elements. We demonstrated that the proposed method achieves very good accuracy and precision in angle measurements as compared with existing approaches.
\end{abstract}

\section{Keywords}

NSGA-II, LMS, PSO, WSN, AoA, PHY, MAC, TDOA,

\section{INTRODUCTION}

A wireless sensor network (WSN) consists of small devices equipped with sensors that can communicate and interconnect wirelessly with each other. These devices are characterized by a constrained battery, memory and processing power. Recently, WSNs have found important application in industrial, military, medical, home, office, and pervasive computing domains. Wireless sensor networks (WSNs) have become progressively more interesting in recent years for the purpose of event monitoring, data acquisition, surveillance, etc. [4][6]Best localization of wireless sensor nodes is a key issue for tiny networks of relatively costly sensing devices. For such networks, the placement problem requires that multiple objectives be met. These objectives are normally incompatible e.g. achieving maximum coverage and maximum connectivity while minimizing the network energy cost. [9]. Smart antenna is the most successful advance for maximum coverage, improved quality and more capacity. Through adaptive beam forming, the base station can form narrower beam toward user that is required signal and nulls toward interfering users that is surplus signals. The weight of antenna arrays can be adjusted to form adaptive beam to track corresponding users automatically by using Algorithms.[10][11] At the same time to minimize interference due to other users, by introducing nulls in the directions of unwanted user. Techniques like LMS, PSO and NSGA-II etc. It is well acknowledged that these techniques are quite successful, reliable and efficient.

The rest of the paper is organized as follows: the next section discusses the basic localization techniques. Section III presents our proposed approach and the corresponding implementation issues. In section IV we present the simulation results and their analysis. Section V concludes the paper.

\section{RELATED WORK}

AoA estimation is a method that decides the direction of arrival of a received signal by processing the signal impinging on an antenna array. Applications of estimating the AoA include beamforming, tracking and localization. The subject of AoA has been extensively studied in the literature. Pantelis K. Varlamos et.al [1] used electronically controllable, switched parasitic planar array for the reason of direction finding. The technique proposed utilizes the electronic beam steering, obtained by simply placing in an appropriate digital word in the antenna feeding circuit, to resolve two signals. A genetic algorithm was used to find out the strengths and the angular positions of the incoming signals in the azimuth plane. The results obtained focuses on wideband signaling in a multipath environment, with and without the presence of noise. The method is mainly reliant on the type of the receiving antenna. Increasing the number of radiation patterns available improves the reliability of the method. The reliability of the method slightly deteriorates, as the angular discrimination of the incoming signals becomes smaller, but the results remain quite satisfying. M. Pastorino et.al. [3] used smart antenna system for the estimation of the directions of arrival (DOAs) of multiple waves. An efficient method based on the support vector regression is proposed, in which the mapping among the outputs of the array and the DOAs of unknown plane waves is approximated by means of a family of support vector machines. For the validation of the proposed approach, multiple impinging waves both in noiseless and 
noisy environments are considered. Minghui Li et. al. [5] proposed an algorithm for bearing estimation in unknown noise fields and harsh WSN scenarios. They established a maximum likelihood (ML) criterion by modelling the noise covariance as a linear combination of known weighting matrices, and a Particle Swarm Optimization (PSO) paradigm is presented for optimization of the ML cost function. Simulation results demonstrate that the paired estimator PSOML significantly outperforms other popular techniques and produces superior bearing estimates, especially in unfavorable scenarios typical of a sensor network. J. Ou Yang et. al. [7] introduced a technique for the optimization of the element excitations of conformal phased array with improved NSGAII (INSGA-II) algorithm Firstly, the patterns of all the elements are measured in a microwave chamber when they are terminated by their own characteristic impedances. Then the pattern of the conformal antenna array can be obtained by a superposition of all these active patterns with various weights. Using the optimization algorithm, the weights including amplitude and phase of each element can be optimized quickly. This method has the advantages of less computation and high accuracy. In order to further accelerate the optimization, INSGA-II algorithm is applied. By using this algorithm, the entire optimal solutions, i.e., the optimal solutions of the main beam of the conformal phased array directing to different angles, can be solved at once. The idea is demonstrated through the designs of two conformal antenna arrays mounted on hemisphere and cylinder-hemisphere platforms, respectively. T.S. Jeyali Laseetha et.al [8] has done realization of SDMA with beam forming and null forming techniques which accounts for co-channel interference rejection. Channel equalization is done using adaptive equalizer to mitigate the effects of ISI. He discussed SDMA technology with digital beam forming with the new idea of including the Variable step-size LMS algorithm along with MUSIC algorithm employed in MAC protocol for IEEE 802.11 and the results of simulation are analyzed for their performance. Nihad A. A. Elhag et.al [12] proposed a new smart antenna signal processing method for estimating the signal angle of arrival (AoA), by using MUSIC method for wideband wireless communications. The simulation is conducted under different random signal arrivals and space between array elements. They showed that the proposed method determined exact angle of arrival of more than $90 \%$ of the received signals Rayleigh fading channel.

Changhwan Choi et.al. [13] proposed user location estimation method that uses smart antenna based reception direction. Existing AoA method is proficient of producing error due to the change of azimuth angle. The range of error increases as the distance between a terminal and base station increases. To solve problem, the method of measuring the reception direction of signal and TOA from several branches can be used. The proposed method is verified by creating same error of azimuth environment by using variety of computer simulations

\section{METHODOLOGY}

We have implemented three different algorithms for AoA estimation as LMS, PSO and NSGA-II.

\subsection{Least Mean Square Algorithm}

This algorithm was first developed by Widrow and Hoff in 1960 .The design of this algorithm was stimulated by the Wiener-Hopf equation. By modifying the set of Wiener-Hopf equations with the stochastic gradient approach, a simple adaptive algorithm that can be updated recursively was developed. This algorithm was later on known as the least- mean-square (LMS) algorithm. The algorithm contains three steps in each recursion: the computation of the processed signal with the current set of weights, the generation of the error between the processed signal and the desired signal, and the adjustment of the weights with the new error information. The following equations summarize the above three steps.

$\hat{d}(n)=w_{1} *(n) u_{1}(n)+w_{2} *(n) u_{2}(n)+\ldots .+w_{t} *(n) u_{t}(n)$

The $\boldsymbol{w}$ in the above equations is a vector which contains the whole set of weights A large step-size allows fast settling but causes poor steady state performance. On the other hand, a small step-size decreases the steady state error but compromises the rate of convergence. The value of parameter is selected by trying out different values in the algorithm.[14]

\subsection{Particle Swarm Optimization}

PSO (Particle Swarm Optimization) is applied to decide optimal antenna elements feed that offer null (minimum power) in the directions of the interfering signals while to maximize of radiation in the direction of the constructive signal. The problem is formulated and resolved by means of the proposed algorithm. Examples are simulated to demonstrate the effectiveness and the design flexibility of PSO.[14]

\subsection{Non-dominated Sorting Genetic Algorithm II (NSGA-II)}

Non-dominated Sorting Genetic Algorithm II (NSGA-II) is a multi-objective genetic algorithm, proposed by Deb et al.[2], in 2002. It is an extension and improvement of NSGA, which is proposed earlier by Srinivas and Deb, in 1995.[2]

In NSGA-II, in addition to genetic operators, crossover and mutation, two specialized multi-objective operators and mechanisms are defined and utilized:

- Non-dominated Sorting: the population is sorted and partitioned into fronts $\left(F_{1}, F_{2}\right.$, etc.), where $F_{1}$ (first front) indicates the approximated Pareto front.

- Crowding Distance: it is a mechanism of ranking among members of a front, which are dominating or dominated by each other.

These ranking mechanisms are used with genetic selection operators, to create the population of next generation.

\subsubsection{Fast Non-dominated Sorting Approach}

As per Deb et.al. [2002] native and slow procedure of sorting a population into different non domination levels and then a fast approach is described.

In a native approach, to determine solutions of the first nondominated front in a population of size $N$, each solution can be compared with every other solution in the population to find if it is dominated. This requires $O(M N)$ comparisons for each solution, where is $M$ the number of objectives. When this process is continued to find all members of the first nondominated level in the population, the total complexity is $O$ $\left(M N^{2}\right)$. [2]

At this stage, all individuals in the first non-dominated front are found. To determine the individuals in the next nondominated front, the solutions of the first front are discounted for the time being and the above procedure is repeated. The task of finding the second front also needs $O\left(M N^{2}\right)$ computations, particularly when $O(N)$ number of solutions belong to the second and higher non-dominated levels. This 
argument is true for finding third and higher levels of nondomination. Thus, when there are fronts and there exists unique solution in each front. This requires overall $O\left(M N^{3}\right)$ computations. Note that storage $O(N)$ is required for this procedure. [2]

First, for each solution calculate two entities: 1) domination count $n_{p}$, the number of solutions which dominate the solution $p$, and 2) $S_{p}$, a set of solutions that the solution dominates. This requires $O\left(M N^{2}\right)$ comparisons.

All solutions in the first non-dominated front will have their domination count as zero. Now, for each solution $p$ with $n_{p}=0$, each member $(q)$ of its set $S_{p}$ and reduce its domination count by one. In doing so, if for any member $q$ the domination count becomes zero, we put it in a separate list $Q$. These members fit in to the second non-dominated front. Now, the above procedure is continued with each member of and the third front is identified. This process continues until all fronts are identified. For each solution in the second or higher level of non-domination, the domination count $n_{p}$ can be at most $N-1$. Thus, each solution $p$ will be visited at most $N-1$ times before its domination count becomes zero. At this point, the solution is assigned a non-domination level and will never be visited again. Since there are at most $N-1$ such solutions, the total complexity is $O\left(N^{2}\right)$. Thus, the overall complexity of the procedure is $O\left(M N^{2}\right)$. Another way to calculate this complexity is to realize that the body of the first inner loop (for each $p \in F_{i}$ ) is executed exactly $N$ times as each individual can be the member of at most one front and the second inner loop (for each q $\in S_{p}$ ) can be executed at maximum $N-1$ times for each individual [each individual dominates $N-1$ individuals at maximum and each domination check requires at most $M$ comparisons] results in the overall $O\left(M N^{2}\right)$ computations. It is important to note that although the time complexity has reduced to $O\left(M N^{2}\right)$, the storage requirement has increased to $O\left(N^{2}\right)$.[2]

In the NSGA-II, replace the sharing function approach with a crowded-comparison approach.

Crowded-Comparison Operator: The crowded-comparison operator $\left(\prec_{n}\right)$ guides the selection process at the various stages of the algorithm toward a uniformly spread-out Pareto optimal front. Assume that every individual $i$ in the population has two attributes:

1. Non-domination $\operatorname{rank}\left(i_{\text {rank }}\right)$;

2. Crowding distance $\left(i_{\text {distance }}\right)$.

We now define a partial order $\prec_{n}$ as:

$$
\begin{aligned}
& \mathrm{I} \prec_{n} \mathrm{j} \text { if }\left(i_{\text {rank }}<j_{\text {rank }}\right) \\
& \operatorname{Or}\left(\left(i_{\text {rank }}=j_{\text {rank }}\right)\right. \\
& \text { And } \left.\left(i_{\text {distance }}<j_{\text {distance }}\right)\right)
\end{aligned}
$$

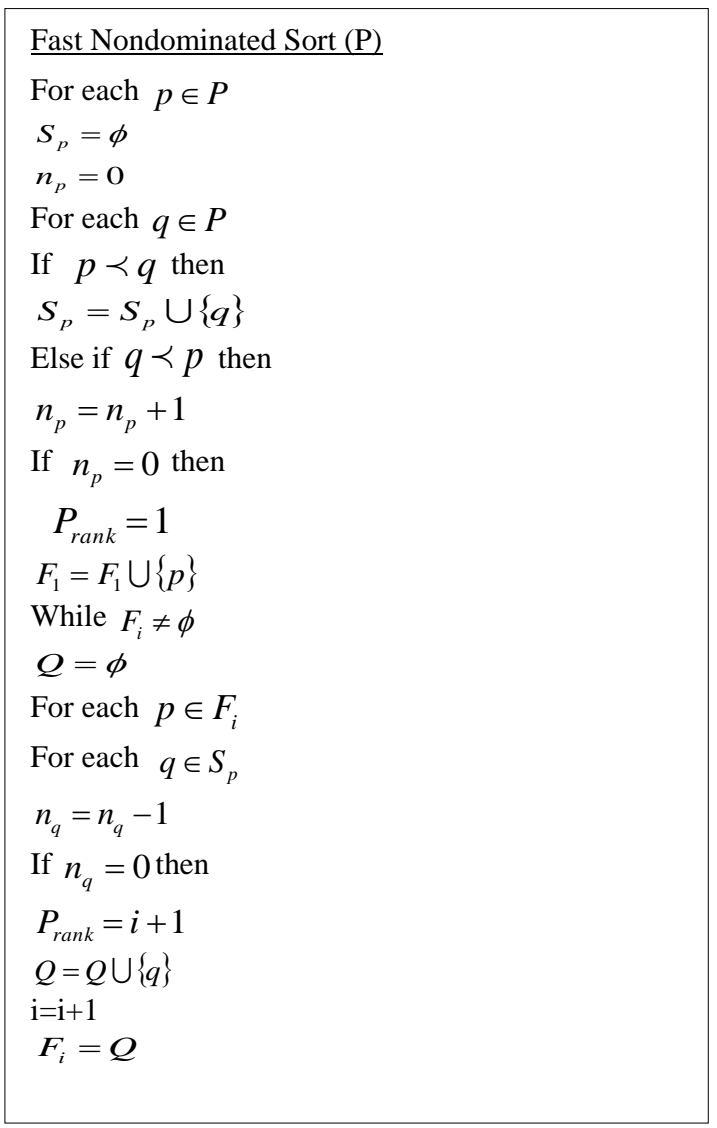

Figure 1: Algorithm for fast Non-dominated Sort [2]

That is, between two solutions with differing non-domination ranks, prefer the solution with the lower (better) rank. Otherwise, if both solutions belong to the same front, then prefer the solution that is located in a lesser crowded region.

With these three new innovations - a fast non-dominated sorting procedure, a fast crowded distance estimation procedure, and a simple crowded comparison operator, describe the NSGA-II algorithm.

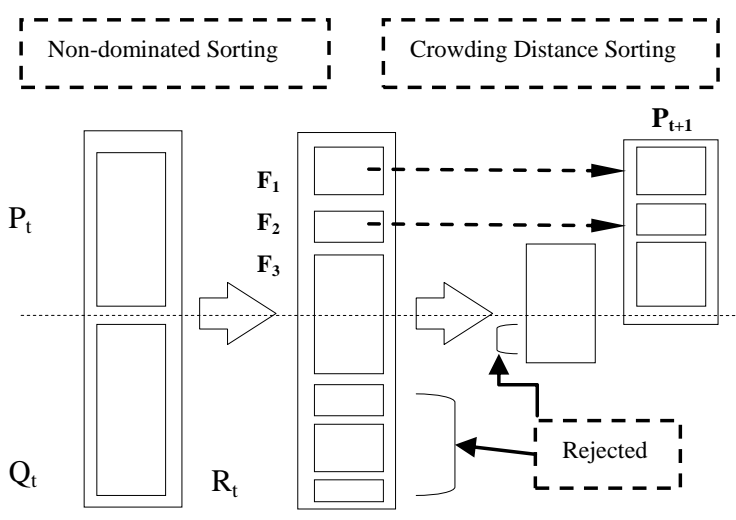

Figure 2: NSGA-II procedure [2].

Initially, a random parent population $\mathrm{P}_{0}$ is created. The population is sorted based on the non-domination. Each solution is assigned a fitness (or rank) equal to its nondomination level (1is the best level, 2 is the next-best level, and so on). Thus, minimization of fitness is assumed. At first, the usual binary tournament selection, recombination, and 
mutation operators are used to create a offspring population $\mathrm{Q}_{0}$ of size $N$. Since exclusiveness is introduced by comparing current population with previously found best non-dominated solutions, the procedure is different after the initial generation.

The step-by-step procedure shows that NSGA-II algorithm is simple and straightforward. First, a combined population $R_{t}=$ $P_{t} \cup Q_{t}$ is formed. The population $R_{t}$ is of size $2 N$. Then, the population $\mathrm{R}_{\mathrm{t}}$ is sorted according to non-domination. Since all previous and current population members are included in $R_{t}$, exclusivity is ensured. Now, solutions belonging to the best non-dominated set $F_{l}$ are of best solutions in the combined population and must be emphasized more than any other solution in the combined population. If the size of $F_{1}$ is smaller than $\mathrm{N}$, choose all members of the set $F_{l}$ for the new population $P_{t+1}$. The remaining members of the population $P_{t+1}$ are chosen from subsequent non-dominated fronts in the order of their ranking. Thus, solutions from the set $F_{2}$ are chosen next, followed by solutions from the set $F_{3}$, and so on. This procedure is continued until no more sets can be accommodated. Say that the set $F_{l}$ is the last non-dominated set beyond which no other set can be accommodated. In general, the count of solutions in all sets from $F_{l}$ to $F_{l}$ would be larger than the population size.

To choose exactly $\mathrm{N}$ population members, sort the solutions of the last front $F_{1}$ using the crowded-comparison operator $\prec_{n}$ in descending order and choose the best solutions needed to fill all population slots. The NSGA-II procedure is also shown in Fig. 1. The new population $P_{t+1}$ of size $\mathrm{N}$ is now used for selection, crossover, and mutation to create a new population $Q_{t+1}$ of size N . It is important to note that we use a binary tournament selection operator but the selection criterion is now based on the crowded-comparison operator. Since this operator requires both the rank and crowded distance of each solution in the population, we calculate these quantities while forming the population, as shown in the above algorithm. Consider the complexity of one iteration of the entire algorithm [2].

$$
\begin{aligned}
& R_{t}=P_{t} \cup Q_{t} \\
& \text { F=Fast non-dominated sort }\left(\mathrm{R}_{\mathrm{t})}\right. \\
& P_{t+1}=P_{t+1} \cup F_{i} \\
& \mathrm{i}=\mathrm{i}+1 \\
& \text { Sort }\left(\mathrm{F}_{\mathrm{i},} \prec_{n}\right) \\
& P_{t+1}=P_{t+1} \cup F_{i} \\
& P_{t+1}=P_{t+1} \cup F_{i}\left[1:\left(N-\left|P_{t+1}\right|\right)\right] \\
& Q_{t+1}=\text { make-new }-\operatorname{pop}\left(P_{t+1}\right)
\end{aligned}
$$

Figure 3: Algorithm of NSGA-II procedure [2]

\section{RESULTS}

The performance of AoA algorithms has been studied in MATLAB environment. For Simulation the following assumptions are considered.

Simulation logarithm: LMS, LMS+PSO and LMS+NSGA-II

AoA of desired signal: $30^{\circ}$

AoA of interference signal: $-60^{\circ}$

\begin{tabular}{|c|c|c|c|c|c|c|c|}
\hline \multirow{3}{*}{$\mathbf{N}$} & \multirow{3}{*}{ d } & \multicolumn{6}{|c|}{ Angle of Arrival (AoA) } \\
\hline & & \multicolumn{2}{|c|}{ LMS } & \multicolumn{2}{|c|}{ LMS+PSO } & \multicolumn{2}{|c|}{ LMS+NSGA-II } \\
\hline & & D.S. & I.S. & D.S. & I.S. & D.S. & I.S. \\
\hline \multirow{3}{*}{4} & 0.25 & $30^{\circ}$ & $-57^{\circ}$ & $28^{\circ}$ & $-55^{\circ}$ & $30^{\circ}$ & $-60^{\circ}$ \\
\hline & 0.5 & $33^{\circ}$ & $-69^{\circ}$ & $33^{\circ}$ & $-67^{\circ}$ & $30^{\circ}$ & $-67^{\circ}$ \\
\hline & 0.75 & $33^{\circ}$ & $-25^{\circ}$ & $33^{\circ}$ & $-25^{\circ}$ & $33^{\circ}$ & $-25^{\circ}$ \\
\hline \multirow{3}{*}{8} & 0.25 & $33^{\circ}$ & $-57^{\circ}$ & $33^{\circ}$ & $-62^{\circ}$ & $30^{\circ}$ & $-60^{\circ}$ \\
\hline & 0.5 & $30^{\circ}$ & $-57^{\circ}$ & $29^{\circ}$ & $-54^{\circ}$ & $30^{\circ}$ & $-60^{\circ}$ \\
\hline & 0.75 & $33^{\circ}$ & $-63^{\circ}$ & $33^{\circ}$ & $-63^{\circ}$ & $33^{\circ}$ & $-63^{\circ}$ \\
\hline
\end{tabular}

Number of data samples: 100

Figure 4 and 5 demonstrates beamplot for various values of number array elements $\mathrm{N}$ and spacing $\mathrm{d}$ considering different algorithms. It depicts how algorithm places adaptively the maxima in the direction of desired user and nulls at AoA of the interferer.

Table 1: AoA estimation for various algorithms

* D.S.:- Desired Signal, I.S.:- Interference Signal

Table 1 infers that LMS+NSGA-II algorithm provides accurate results with increase in number of elements $\mathrm{N}$ of the array. If the spacing $\mathrm{d}$ between array elements increased towards unity then the results deviates by $3^{\circ}$ with more value of N.Less value of $\mathrm{N}$ with more value of $\mathrm{d}$ deviates interference signal angle keeping proper desired signal angle.

LMS and LMS+PSO algorithm delivers AoA of either D.S. or I.S., but not both at a time for various values of $\mathrm{N}$ and $\mathrm{d}$.

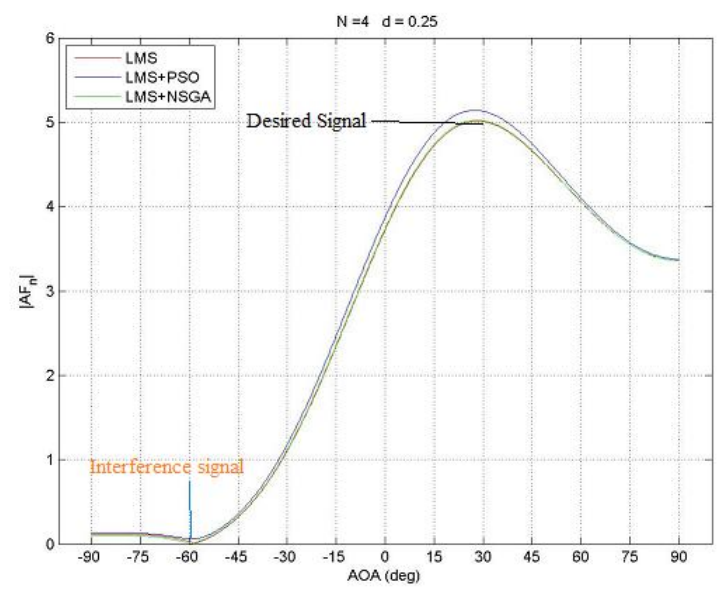

Figure 4 a) Beam Plot for $\mathrm{N}=4, \mathrm{~d}=\mathbf{0 . 2 5}$ 


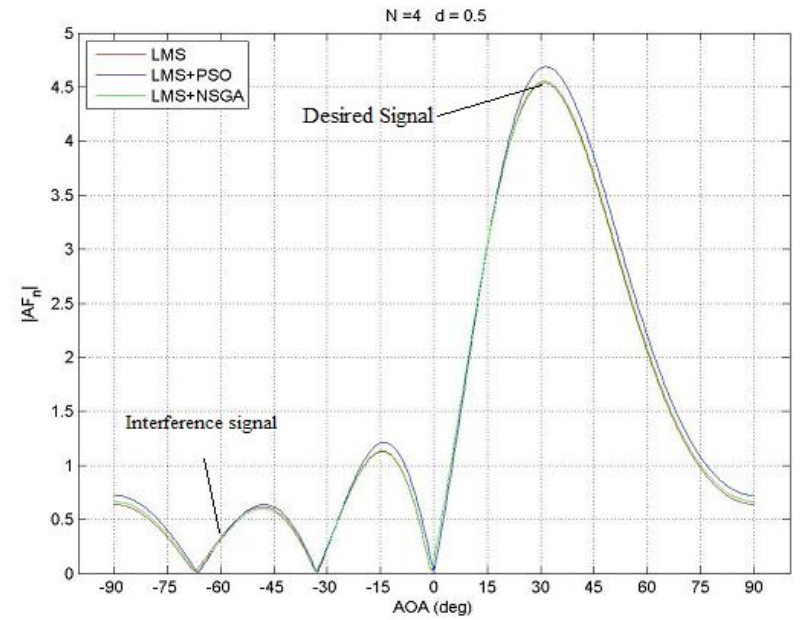

Figure 4 b) Beam Plot for $N=4, d=0.5$

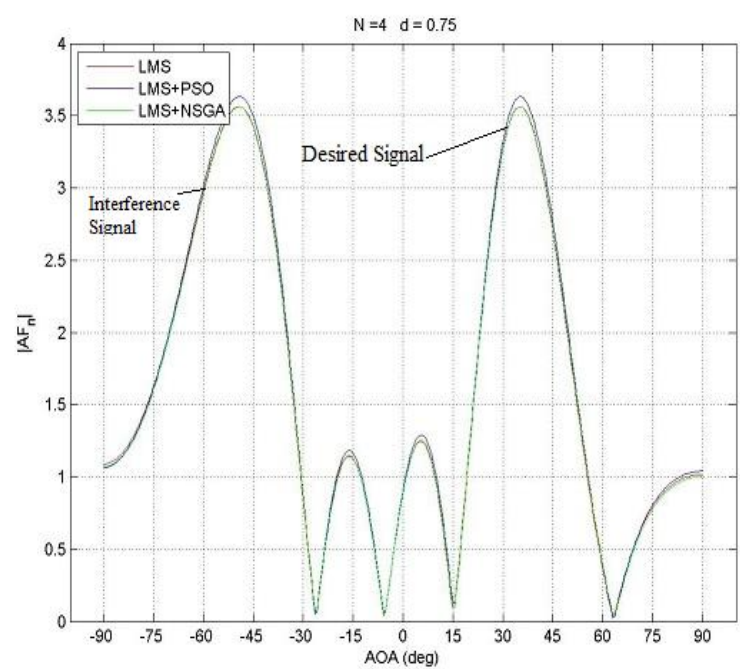

Figure 4 c) Beam Plot for $N=4, d=0.75$

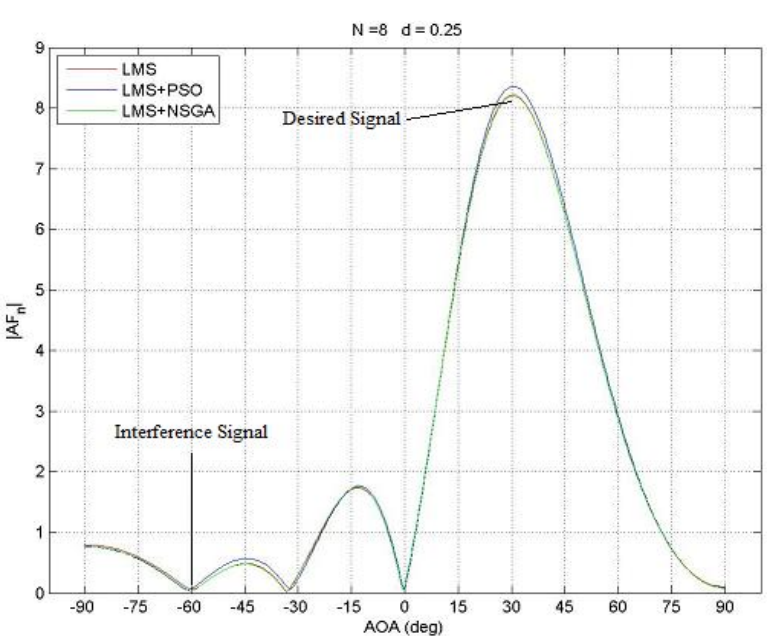

Figure 5a ) Beam Plot for $\mathrm{N}=8, \mathrm{~d}=\mathbf{0 . 2 5}$

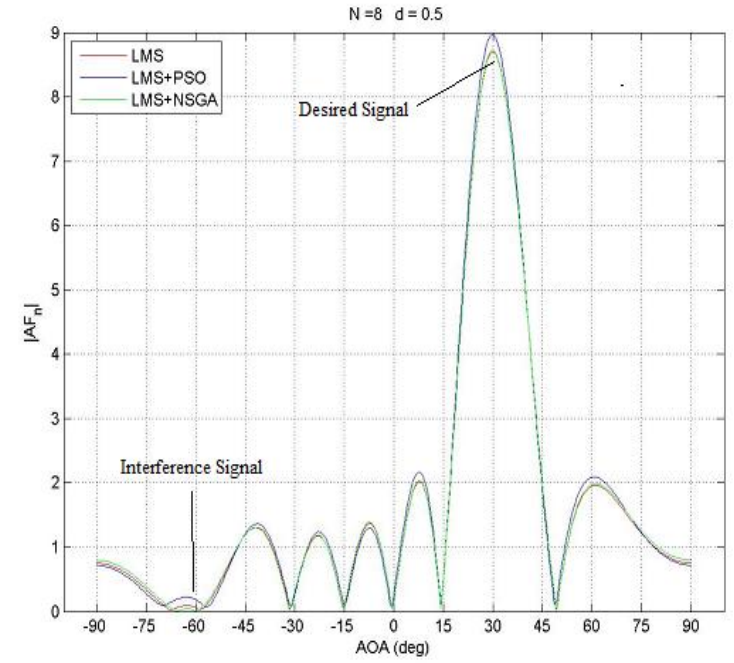

Figure 5b ) Beam Plot for $\mathrm{N}=8, \mathrm{~d}=0.5$

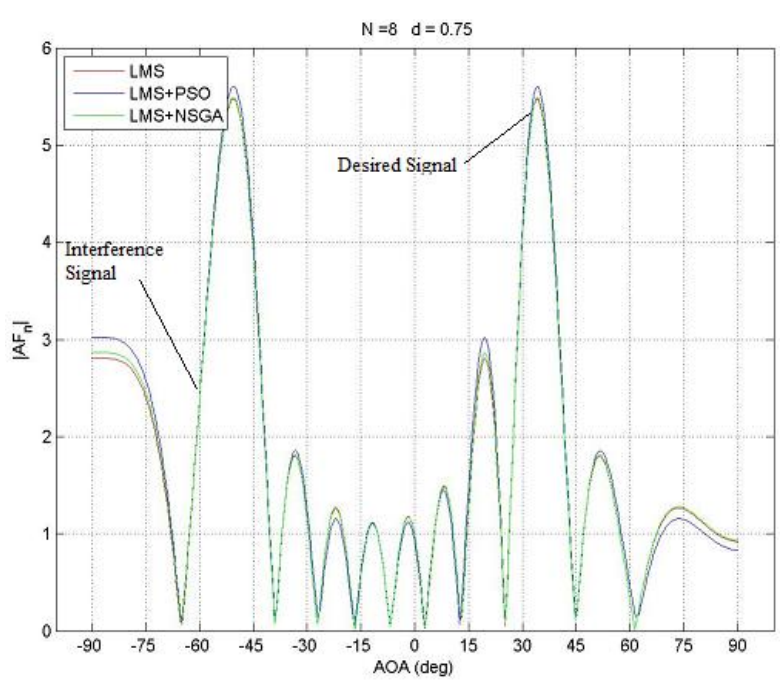

Figure 5c ) Beam Plot for $\mathrm{N}=8, \mathrm{~d}=\mathbf{0 . 7 5}$

\section{CONCLUSION}

In this paper, we presented a NSGA-II optimization alogirthm applied for estimation of AoA for wireless sensor networks. Three different algorithms have been implemented by vaying number array elements and it's spacing. Weights achieved by LMS algorithm is further optimized using PSO and NSGA-II for getting beamforming in the desired direction and reducing the interference. The simulation results show that AoA measurements is improved with the our proposed approach LMS+NSGA-II which exhibits very good accuracy and precision. This may result in better localization coverage, efficient routing and better data reception.

\section{REFERENCES}

[1] Pantelis K. Varlamos and Christos N. Capsalis, Direction-of-Arrival Estimation (DoA) Using Switched Parasitic Planar Arrays and the Method of Genetic Algorithms, Article in Wireless Personal Communications 28(1):59-75 - January 2000

[2] K. Deb, A. Pratap, S. Agarwal, and T. Meyarivan, "A Fast and Elitist Multi-objective Genetic Algorithm: 
NSGA-II," IEEE Trans. Evol. Comp., vol. 6, no. 2, Apr. 2002, pp. $182-197$

[3] M. Pastorino ; A. Randazzo , A smart antenna system for direction of arrival estimation based on a support vector regression, IEEE Transactions on Antennas and Propagation (Volume:53 , Issue: 7 ) pp:2161 2168,July 2005

[4] Rong P and Mihail L. Sichitiu, Angle of Arrival Localization for Wireless Sensor Networks, 3rd Annual IEEE Communications Society on Sensor and Ad Hoc Communications and Networks (Volume:1 ), pp:374 382,Sept. 2006

[5] Minghui Li ; Yilong Lu ,Angle-of-arrival estimation for localization and communication in wireless networks, 2008 16th European Signal Processing Conference, Pp:1 -5 , Aug. 2008

[6] Adnan Kavak ; ; Kerem Kucuk, On connectivity analysis of smart antenna capable wireless sensor networks, 2009 6th International Symposium on Wireless Communication Systems , pp: 6 - 10Sept. 2009

[7] J. Ou Yang , Q.R. Yuan, Fair Yang,H.J. Zhou, Z.P. Nie,Zi-qin Zhao, Synthesis of Conformal Phased Array With Improved NSGA-II Algorithm Article in IEEE Transactions on Antennas and Propagation 57(12):4006 4009 . January 2010

[8] T.S. Jeyali Laseetha and R.Sukanesh , Smart AntennaAOA Estimation Employing Music Algorithm And Digital Beamforming By Variable Step-Size LMS Algorithm With Novel Mac Protocol For IEEE 802.11, ICNVS'10 Proceedings of the 12th international conference on Networking, VLSI and signal processing, pp: 187-192, 2010
[9] F. Zaman1,I. M. Qureshi , A. Naveed, J. A. Khan , and R. M. A. Zahoor, Amplitude And Directional Of Arrival Estimation: Comparison Between Different Techniques, Progress In Electromagnetics Research B, Vol. 39, 319335,2012

[10] E. D. Skiani ; S. A. Mitilineos ; S. C. A. Thomopoulos, A Study of the Performance of Wireless Sensor Networks Operating with Smart Antennas, IEEE Antennas and Propagation Magazine (Volume:54 , Issue: 3 ),pp:50-67, 2012

[11] Tanuja S. Dhope ,Dina Simunic ; Nikhil Dhokariya ; Vishal Pawar; Bhawana Gupta, Performance analysis of angle of arrival estimation algorithms for dynamic spectrum access in cognitive radio networks, 2013 International Conference on Advances in Computing, Communications and Informatics (ICACCI), ,pp:121 - 126, Aug. 2013

[12] Nihad A. A. Elhag; Isam M. Osman; Alaa A. Yassin ; Tsneem B. Ahmed, Angle of arrival estimation in smart antenna using MUSIC method for wideband wireless communication, 2013 International Conference on Computing, Electrical and Electronics Engineering (ICCEEE), Pp :69 - 73,Aug. 2013

[13] Changhwan Choi ; Yongwan Park; Soojung Hur, Location estimation algorithm using smart antenna, 2014 International Conference on Indoor Positioning and Indoor Navigation (IPIN), pp:753 - 755,Oct. 2014

[14] Nayan B. Shambharkar, P. M. Mainkar,G. N. Mulay,Optimization Techniques for Smart Antenna Beamforming, International Journal of Mobile \& Adhoc Network, Vol 3, issue 4, November 2013 